
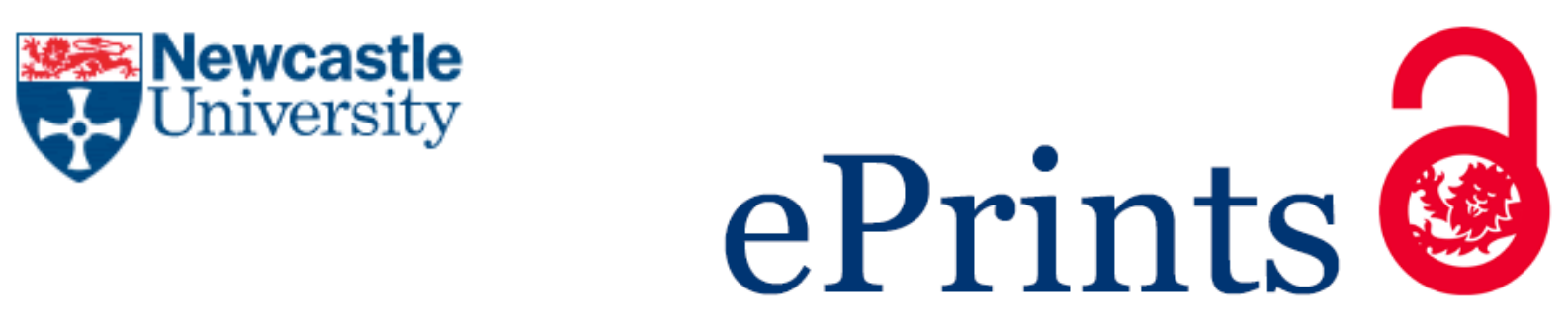

Mullen C, Wade NS, Olabisi O, Taylor PC.

The impact of the coincidence of STOR and triad events on STOR provider's net-income considering load recovery characteristics.

In: CIRED Workshop. 2016, Helsinki, Finland: Institution of Engineering and Technology.

\title{
Copyright:
}

Copyright IET, 2017. This is the author's manuscript of a paper that was presented at CIRED Workshop Conference, held 14-15 June 2016, Helsinki, Finland.

DOI link to article:

http://doi.org/10.1049/cp.2016.0800

Date deposited:

$04 / 05 / 2017$ 


\section{THE IMPACT OF THE COINCIDENCE OF STOR AND TRIAD EVENTS ON STOR PROVIDER'S NET-INCOME CONSIDERING LOAD RECOVERY CHARACTERISTICS}

\author{
Christopher MULLEN \\ Newcastle University - UK \\ c.mullen@ncl.ac.uk
}

\author{
Neal S. WADE \\ Newcastle University - UK \\ neal.wade@ncl.ac.uk
}

\author{
Ola OLABISI \\ Siemens - UK
}

\author{
Philip C. TAYLOR \\ Newcastle University - UK
}

\begin{abstract}
Demand side response (DSR) providers must consider several factors when participating in grid services by demand reduction. Considering the GB network, this investigation analyses the net income probabilities due to the coincidence of DSR calls for system balancing with peak demand charges, and the influence of demand recovery characteristics on this. This work develops a statistical model of STOR based on published market data and a demand model that calculates the Triad charge and the income from STOR provision, as well as the distribution use of system (DUoS) charges and energy costs. Using these models with historical Triad data, this paper illustrates the statistical spread of financial revenue to the STOR provider given the timing of STOR and Triad periods. The model is also used to investigate the influence of demand recovery shapes on the provider's net income. This can be used in assessing whether demand recovery shape is an important consideration for the provider's DSR strategy.
\end{abstract}

\section{INTRODUCTION}

In order to compensate the GB transmission network owner for peak electricity demand the GB network use of system charges includes a peak demand charge to half-hourly metered customers known as Triad. Triad periods represent the three peak half-hours of the Winter season, November to February. They may be considered as a form of critical peak-pricing, however the Triad periods are not known in advance but are calculated after the Triad season. For half-hourly metered customers, demand during these periods represents a significant additional cost on their electricity bill [1]. For example the Triad charge in the Northern region was $22.346537 \mathrm{E} / \mathrm{kW}$ in 2013-14 [2]. Some customers reduce their demand during forecast Triad periods to minimize their exposure to Triad costs. This is known as "Triad avoidance".

The GB transmission system operator procures short term operating reserve (STOR) as part of a suite of mechanisms to maintain system balance. STOR is designed to mitigate imbalance with a response time from minutes to hours and can be provided from increased generation or demand reduction. It is comparable with "tertiary reserve" services in other countries since it can be called off manually and the response is usually on a time scale of minutes [3] although technically it can be up to 4 hours [4]. An electricity demand customer can gain availability and utilization income by offering a STOR service by demand reduction. However, providers which are not part of the balancing mechanism must also pay for the required metering [5]. The minimum power provision is 3 MW but smaller providers can participate via an aggregator. STOR provision is an exclusive service and providers may not engage in other forms of demand side response, including “Triad avoidance” [4].

Electricity consumers that reduce demand significantly may see a temporary increase in demand above the expected level if there had been no demand reduction. This load recovery effect can be due to demands with inherent storage (for example space heating/cooling). The occurrence and shape of recovery will depend on the type of loads which have been reduced.

An electricity user providing STOR by demand reduction may benefit from reduced Triad charges if the demand reduction due to a STOR call coincides with the Triad period. However Triad charges may be increased if load recovery coincides with a Triad period.

This work develops object-oriented MATLAB models to explore the probabilities of Triad and STOR coincidence and it considers demand recovery to assess the potential financial impact on a STOR provider. Modelling is based on data from the year $2013-14$.

\section{STOR AND TRIAD COINCIDENCE 2013-14}

STOR is called within pre-defined time windows which vary by day-type (working/non-working day) and time of year. All the Triad periods in 2013-14 were between 17:00 to 17:30. For the windows which would coincide with a Triad the earliest window time for 2013-14 is 16:00. Figure 1 illustrates the maximum Triad demand reduction due to a STOR call.

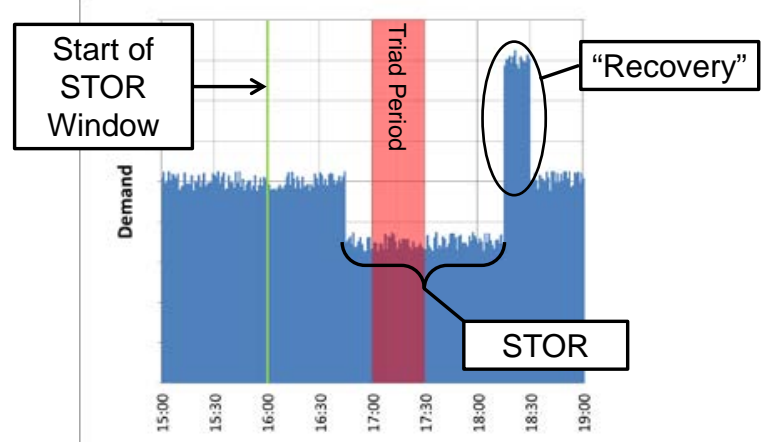

Figure 1 Illustration of STOR and Triad coincidence 
For loads without demand recovery (and including STOR provision by Diesel generator) STOR and Triad coincidence can only reduce the Triad demand. However, for loads with energy recovery, the change in Triad demand due to STOR provision depends the demand recovery shape and on the time and duration of the STOR call. Considering the STOR call:

- For a STOR duration of 1.5 hours the demand reduction will always totally overlap the Triad period providing the STOR call is prior to the Triad period, since the earliest start time would be at 16:00. In this case the load recovery period would always be after the Triad.

- For STOR calls less than 1.5 hours there is a chance that any load recovery would occur during the Triad period, depending on the combination of STOR call time and duration.

If the demand recovery coincides with any part of the Triad period, the increased Triad demand depends on the duration for which the recovery peak coincides with the Triad period and the average power over that duration.

\section{MODELLING}

Modelling was achieved using MATLAB Object Oriented Programming. A time resolution of 1 minute was used to give sufficient resolution to the overlap time of events within the 30 minute Triad period. Triad periods and DUoS charges from 2013-14 are used in the modelling $[6,7]$.

\section{STOR model}

The STOR model is based on data given in [3]. The first stage is to determine whether a STOR call occurs on the day under consideration. If it does then the time of the call is determined. For the purpose of this work the duration of a STOR call is a control parameter.

\section{Determining if the day is a STOR day}

The STOR energy utilised, available in [3, 8], is different in each season. These values can be used as a proxy for the probability of a STOR call in that season :

$$
P_{S}=\frac{E_{S}}{E_{\text {Total }}} \times N_{\text {Total }}
$$

where $E_{S}$ is the energy utilized in season $S$;

$E_{\text {Total }}$ is the total energy used over the year; $N_{\text {Total }}$ is the total number of calls over the year.

The value for the expected total number of calls in a STOR year was set at 60 .

Paper No 0365
The probability of a call on a particular day is given by:

$$
P_{D}=\frac{P_{S}}{n_{\mathrm{DS}}}=\frac{E_{S}}{E_{\text {Total }}} \times \frac{N_{\text {Total }}}{n_{\mathrm{DS}}}
$$

where $n_{\mathrm{DS}}$ is the number of days in a season

\section{Determining the time of a STOR call}

STOR energy called for each half-hour period on different days of the week and in different STOR seasons is given in $[3,8]$. This data was used to produce a cumulative curve to give the time of the call.

\section{Load Model}

The assumed demand is a constant $800 \mathrm{~kW}$ except for STOR calls, when it is reduced by $100 \mathrm{~kW}$. The motivation for using constant demand is to isolate the influence of STOR duration and energy recovery shape on the cost benefit.

Load recovery is assumed to be $100 \%$ of the energy reduced during demand reduction and the shape is characterised in the model by the recovery duration. A shorter duration will give a recovery with a larger peak.

\section{Electricity bill and STOR income models}

A model of the electricity bill includes energy cost, Triad and DUoS charges. The income from STOR assumes an availability price of $4.94(£ / \mathrm{MW}) / \mathrm{h}$ and a utilization price of 183.76 ( $£ / \mathrm{MWh}$ ) which are average values when long term contracts have been removed [3]

\section{METHOD}

To generate each data point the model first calculates the electricity bill over the Winter season for a nonSTOR provider. Then the bill and STOR income with STOR demand response is calculated 10,000 times and each result is compared to the non-STOR provider bill to give a cost-benefit of providing STOR. The relative cost benefit is related to the bill for a non-STOR provider :

$$
B_{\mathrm{rel}}=1+\frac{S_{\mathrm{inc}}-B_{\mathrm{STOR}}}{B_{\text {NONSTOR }}}
$$

where $S_{\text {inc }}$ is the total STOR income; $B_{\mathrm{STOR}}$ is the bill cost for a STOR provider; $B_{\text {NONSTOR }}$ is the bill cost for a non-STOR provider.

\section{RESULTS}

Figure 2 shows the mean increased in Triad demand over 10,000 runs relative to the non-STOR Triad demand. There is no chance of negative cost benefit if there is no energy recovery. Also there can be no energy

Page 2 / 4 


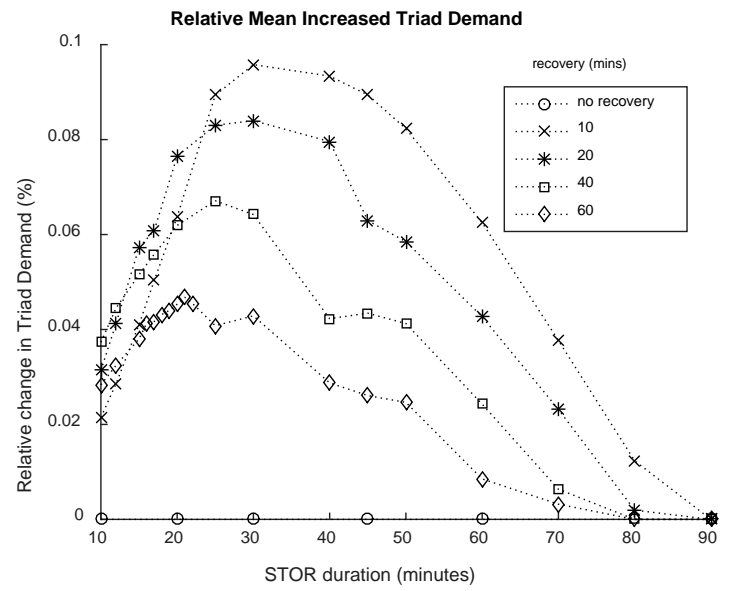

Figure 2 Mean increase in Triad demand

recovery during Triad for STOR durations of 90 minutes or longer so there is no chance of increased Triad demand.

For STOR durations greater than one hour the only case where load recovery would coincide with Triad is if the STOR call started in period 33 (16:00 - 16:30). However there is significantly more STOR energy procured in period 34 (16:30- 17:00) meaning that a STOR call is less likely in period 33. As STOR durations move to values less than an hour there is a increased chance of an energy recovery during Triad due to a call starting in period 34. This is why the probability of increased Triad demand is higher for STOR durations less than 60 mintes compared to the 60 -90 minute range.

For STOR durations less than 20 minutes the demand response energy is smaller resulting in a smaller demand recovery energy and therefore a lower recovery peak.

Figure 3 and Figure 4 show examples of the cost benefit factor probability histograms for STOR durations of 30 and 90 minutes and equivalent energy recovery duration (10 minutes). As the STOR duration increases the peak probability occurs at a higher cost benefit due to the increased STOR utilization income. The probability spread also becomes wider. For 30 minute duration there are some occurrences of negative cost benefit, whereas there are none for 90 minute STOR duration.

Figure 5 shows STOR duration versus mean cost benefit. For the same STOR duration results are within about $£ 30$ for differing rebound times except that the no load recovery case has an income around $£ 100$ higher, based on $100 \mathrm{~kW}$ demand reduction and using the electricity costs and STOR income values stated earlier

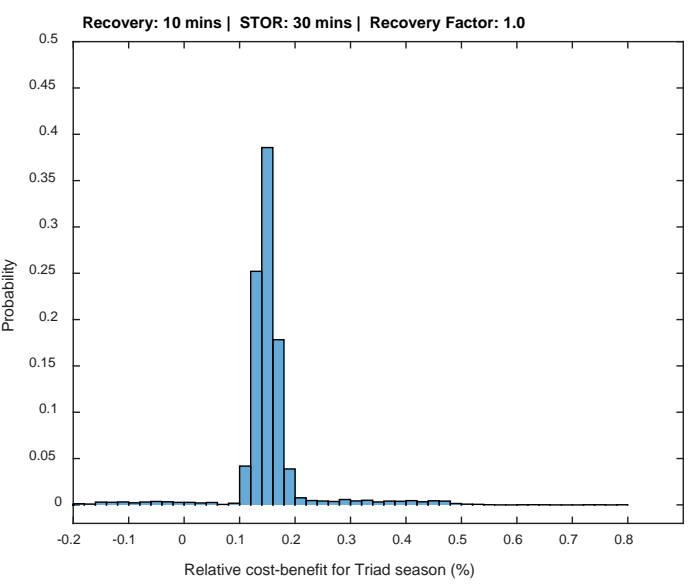

Figure 3 Cost benefit for Triad season for 30 minute STOR and 10 minute load recovery

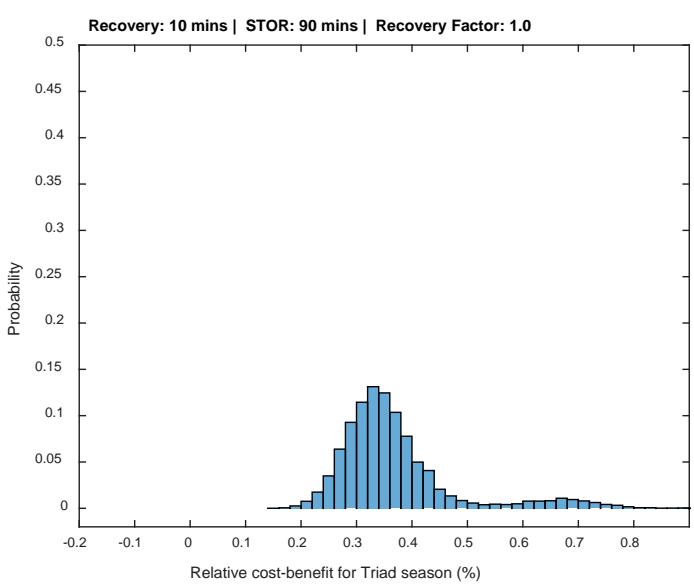

Figure 4 Cost benefit for Triad season for 90 minute STOR and 10 minute load recovery

in the section on modelling.

Whilst the influence of non-zero load recovery duration only makes a difference of up to $£ 30$ for the mean cost benefit, it's influence at lower probability events is more significant. Figure 6 shows the probability of negative cost benefit for the model. In general the probability of negative cost benefit increases as the STOR duration and rebound duration decrease. However at very short duration STOR calls the probability reduces.

\section{DISCUSSION}

This work indicates that there is a risk of increased Triad charges to a STOR provider using demand reduction if the demand has energy recovery. The extent of the increased charges depends more on the whether 


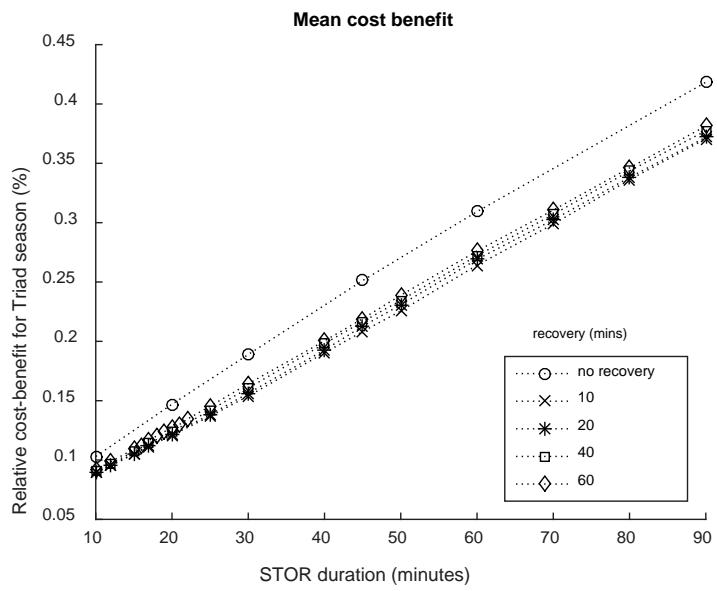

Figure 5 Mean cost benefit

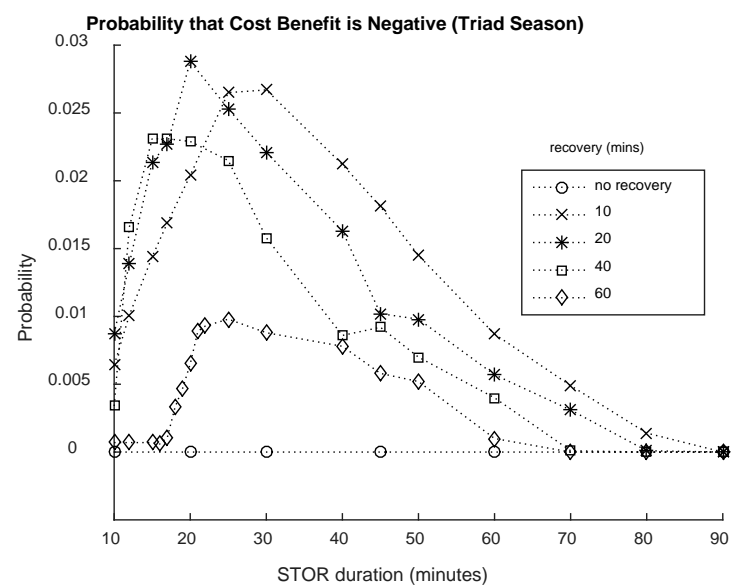

Figure 6 Probability of negative cost-benefit (Triad season)

there is energy recovery than the shape of the recovery. This may result in providers re-assessing their approach STOR provision.

The modelling described in this paper only applies to the Triad periods and STOR data for 2013-14. Although Triad periods commonly occur at 17:00 - 17:30 they may be different in other years which would influence the probability curves. In addition, the prices for STOR have been reducing and this will affect the financial benefit achievable. The work also does not consider the capital outlay in metering equipment required for STOR providers

A standby Diesel generator could be used to provide STOR which would avoid any additional Triad charges due to energy recovery. However this would incur capital costs for grid connection; fuel, refuelling and maintenance charges and would generate $\mathrm{CO} 2$ and other emissions [1] the costs of which are not considered in Paper No 0365 this work.

The Winter period is considered, however STOR income is available all year round. Whilst this may offset any loses due to energy recovery and Triad interaction, a STOR provider is able to opt out at up to a week ahead and may prefer to do this during the Winter months.

The STOR durations in the modelling are fixed because they are used as a control parameter, however this causes a weakness in the modelling. Considering a STOR call of 30 minutes which results in energy recovery during a Triad period the modelled STOR utilization for the rest of the Winter season is based on fixed 30 minute calls. However for an actual STOR provider the STOR durations will be of varying length and are likely to be greater than 30 minutes. This means that the utilization income is likely to be higher than the model would suggest and this would increase the cost benefit.

\section{REFERENCES}

[1] C. Mullen, P. C. Taylor, V. Thornley, and N. S. Wade, "Use of standby generation for reduction of transmission network charges for half-hourly metered customers," in Power Engineering Conference (UPEC), 2014 49th International Universities, 2014, pp. 1-6.

[2] National Grid, "The Statement of Use of System Charges - Effective from 1st April 2013," 2013.

[3] National Grid, "Short Term Operating Reserve Annual Market Report 2013-14," 2015.

[4] National Grid "Short Term Operating Reserve (STOR) Frequently Asked Questions " Nov 2013.

[5] National Grid, "Short-term operating reserve despatch procedure "STOR despatch procedure" for Non-Balancing Mechanism Participants," 2008.

[6] National Grid, "2013 - 14 Triad Data," ed. http://www2.nationalgrid.com/UK/Industryinformation/System-charges/Electricitytransmission/Transmission-Network-Use-ofSystem-Charges/Transmission-Charges-TriadData/, 2014, p. 2.

[7] Northern Powergrid, "Statement of Use of System Charging (LC14) Final Notice Effective 1 April 2013," 2013.

[8] National Grid, "STOR Annual Market Report 2013-14 Data (Excel)," ed, 2015. 